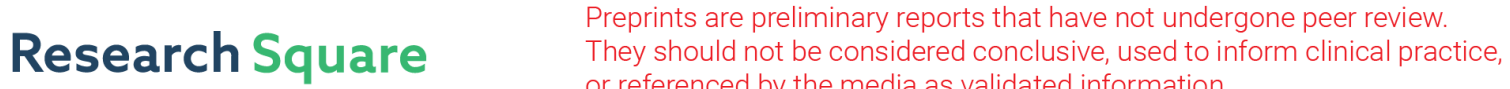 or referenced by the media as validated information. \\ CEBM: Collaborative Ensemble Blockchain Model for Intrusion Detection in loT Environment
}

\section{Omkar Shende}

Maulana Azad National Institute of Technology

R. K. Pateriya

Maulana Azad National Institute of Technology

Priyanka Verma ( $\nabla$ 303priyanka.verma@gmail.com )

Atal Bihari Vajpayee Indian Institute of Information Technology and Management

https://orcid.org/0000-0002-2153-893X

Anupama Jain

Maulana Azad National Institute of Technology

\section{Research Article}

Keywords: CIDS,IoT,Ensemble model,Blockchain,Anomaly detection

Posted Date: July 20th, 2021

DOI: https://doi.org/10.21203/rs.3.rs-702181/v1

License: (c) (1) This work is licensed under a Creative Commons Attribution 4.0 International License. Read Full License 


\title{
CEBM: Collaborative Ensemble Blockchain Model for Intrusion Detection in IoT Environment
}

\author{
Omkar Shende $^{1}$. R. K. Pateriya ${ }^{1}$. \\ Priyanka Verma $^{1}$. Anupama Jain ${ }^{1}$
}

\begin{abstract}
In recent years, the IoT environment devices have increased drastically, including smart home devices, ad-hoc networks for the automotive industry (VANET), healthcare, smart cities, and apparel. In today's IoT environment, security is a critical topic due to the type of devices, the nature of the architecture, different communication techniques (primarily wireless), and amount of information shared over the network. Unfortunately, due to this diverse nature of IoT devices it is very difficult to analyze the behavior of intrusion traffic, as it suffers from ability of recognizing new attacks. Another issue is that the poisoning attack during training of model as intruder may inject malicious data as benign during training of model to create a backdoor for intrusion. Moreover, it is very difficult to create a good intrusion detection dataset which contains all kind of traces. Therefore, to protect the network including IoT, from various threats and attacks, Intrusion Detection Systems (IDSs) are among the best security methods that can be used. However, an isolated IDS node cannot overcome the problems. Therefore, different IDS need to collaborate in order to improve their performance. Thus, in this paper we propose a generic framework of collaborative ensemble blockchain model (CEBM) for intrusion detection in IoT environment. The proposed framework enables the IDS modules to securely share information and build an ensemble detection model by combining the different machine learning models with the best performance using blockchain in a confirmable approach in the absence of a trusted intermediary. UNSW_NB15 dataset is used to evaluate the proposed framework, and five different IDS modules are trained. The performance achieved by the col-
\end{abstract}

Omkar Shende

E-mail: shendeomkar195@gmail.com

R.K. Pateriya

E-mail: pateriyark@manit.ac.in

Priyanka Verma

E-mail: 303priyanka.verma@gmail.com

Anupama Jain

E-mail: jain.anupama.vds@gmail.com

${ }^{1}$ Maulana Azad National Institute of Technology (MANIT), Bhopal, India 
laborative ensemble model is presented in the form of accuracy, recall, precision, and F-score given as $99.113 \%, 99.177 \%, 99.559 \%$, and 0.9942 , respectively.

Keywords CIDS · IoT · Ensemble model · Blockchain · Anomaly detection

\section{Introduction}

The Internet of Things (IoT) is considered to be the next stage in the development of the Internet [3], due to the rapid growth and spread of the IoT and has become the main feature of our daily life [2]. The IoT environment consists of multiple sensors and devices that are interconnected to one another and the internet. These devices or sensors record environmental data and share it [1]. The IoT environment offers efficient resource management and ubiquitous coverage, making it widely used in many different areas such as smart cities, healthcare, smart grids, etc. [9]. According to a report by Statista, the total number of devices in the IoT is overgrowing every year and is expected to reach 75.44 billion devices by 2025 [8]. IoT security is becoming increasingly complex with the growth and expansion of the industry. Moreover, due to the variety of IoT architectures, different types of accessible devices, and the large amount of data being sent over the network, [4-7] are also the reason for IoT security.

To achieve this, Intrusion Detection Systems (IDS) is the primary and critical security mechanism for protecting IoT environments from security threats [10]. Traditionally, IDS can be divided into two parts depending on the detection method as signature-based IDS and anomaly-based IDS.

Anomaly-based IDS attacks are detected by comparing the previously known normal traffic profile with current traffic. If the traffic deviates from the normal traffic profile, it is classified as anomaly traffic. Even though it is used in a wide range of IDS, this method suffers from a large number of false-positive alarms but can detect zero-day attack [11-13]. On the other hand, signature-based IDS compares the traffic with the signature of the previously known attacks; for this purpose, a database of attack signature is created. Signature-based IDS has a low false-positive rate, but it cannot detect zero-day attacks [14]. In both cases, performance can be improved by either building a strong normal traffic profile or increasing the signature database size. This can be done by a collaboration of different IDS modules, where under a certain set of rules, other IDS can share their knowledge for the improvement of overall performance.

Moreover, to detect DDoS attack Verma et al. [33] formulated an attack equation to identify the attack VMs in the network. A bio-inspired Cuckoo search technique mentioned in [37] uses bivariate flight to label the attack and benign request. However, both the methods are unable to detect the zero day attack.

Even though collaboration of IDS modules seems beneficial to improve performance and detection rate, but it suffers from various challenges like Secure connection, centralization, trust management, transparency, etc. [16]. Combining Collaborative IDS modules with blockchain can overcome the challenges mentioned above. Blockchain is a secure, reliable, and decentralized database or general ledger account distributed over the network and contains all transactions in a digital structure [15]. Blockchain is just a data structure connected with encrypted values called the hash. The main elements of blockchain are previous hash value, current 
hash value, the hash value of root block, random number for consensus process (Nonce), timestamp, and data or information. However, the block structure can be changed according to the personal requirement or need.

The block content cannot be altered without altering all the subsequent blocks as the hash value of the block is calculated based on the combination of the block information and previous hash $[17,18]$. Based on the specific type of permission control, existing blockchain technology can be categorized as Public, Private, and Consortium. A blockchain is known as a public blockchain if it enables a person to act as an unrestricted reader and writer like Bitcoin [19] and Ethereum [20]. The consortium blockchain allows only a small group of entities to read or write to the blockchain, which is registered and verified by the system. On the other hand, a private blockchain is often managed by a single entity but can be deployed in different locations. The consensus protocol is an integral part of the blockchain system, responsible for keeping the blockchain up to date and ensuring that all stakeholders involved agree on a unified view of the book. It depends on the implementation of the blockchain, and the threat model [21]. Proof of work, proof of stake, and proof of elapsed time are consensus protocol types.

Proof of work: In this consensus protocol, a node can accept a block if there is proof that a specified computational effort or workload has been expended.

Proof of stake: This method provides a consensus-based on the random selection and impact of a participant (also called stake). It is believed that the block integrity of entities is ensured when the stakes are high.

Proof of Elapsed time: An entity requests each potential validator to share a safe and random wait time from a trusted environment to ensure consensus is achieved.

\subsection{Contributions}

The main contributions of the research include:

- The proposed CEBM shares the trained model with its peers using blockchain, ensuring that the data cannot be tamper and always be secure. Moreover, blockchain is also used to track each IDS module's contribution and is rewarded with digital coins that can be used only within the IDS network to use/buy resources from its peers.

- The proposed framework helps to improve the performance of IDS by collaborating with other IDS and solves the trust and security issues present in traditional collaborative IDS with blockchain technology.

- Additionally, sharing of trained model and resources is very important for newly developed IDS modules, as the new IDS might not have a strong dataset or information to train their model to achieve good performance.

- On the other hand, in the IoT environment, some of the services or devices are rarely used. There is limited data available, which might cause poor performance or overfitting, which can be solved by a collaboration of IDS modules using blockchain.

The rest of the paper is organized as: Section 2 presents the related work; Section 3 describes the proposed work in detail, Section 4 shows the experimental 
results using the proposed framework, and finally, Section 5 derives the conclusion of the proposed framework.

\section{Related Work}

Many researchers have designed a framework for the collaboration of different IDS modules using other protocols and techniques.

The objective of Wenjuan Li et al. [22] is to create a decentralized and distributed signature database on blockchain to increase the performance and robustness of intrusion detection and to avoid insider attacks in collaborative IDS networks. For this purpose, a generic approach is developed that can create a database with trusted signatures in a collaborative IoT environment and update it gradually.

Rathore et al. [9] proposed a decentralized security architecture based on a software-defined network (SDN) combined with blockchain technology for IoT networks in the smart city to solve monitoring problems existing security mechanisms and collecting historical data in the IoT network.

The proposed model by Manikumar et al. [24] detects DDoS attacks using different machine learning models such as KNN, Decision tree, and random forest, from which Random forest has the best performance of $95 \%$ accuracy. After detecting the attack, the IP address of the attacker is stored on the blockchain. In this proposed method, blockchain is used as a blacklist of IP address; whenever an attack is detected, the attacker's IP address is added to this and is blocked for a specific time frame.

Meng et al. [23] designed a collaborative architecture for the IDS environment to secure the collaborative network from insider attacks. An enhanced challengebased trust management method using blockchain is proposed to tackle insider attacks. The method is evaluated using both simulated data and real-time data. It is found that the proposed framework is even effective for advanced insider attacks such as passive message fingerprint attacks (PMFA).

$\mathrm{Li}$ et al. [15] presents the different application and use cases of blockchain and its advantages and implementation issues in the current scenario. The article proposes an information security model based on blockchain intrusion detection technology in the IoT environment. Intrusion detection technology helps protect information security in the blockchain and analyzes various models based on intrusion detection technology.

A lightweight collaborative framework is proposed by Mirsky et al. [2] with the blockchain, and the trust anomaly model is gradually updated with self-assessment and consensus among IoT devices. Raspberry pi is used to evaluate the proposed framework with a variety of IoT services. The proposed framework effectively detects various types of attacks without false alarms.

The purpose of literature Li et al. [25] is to design a CIDN framework based on a blockchain challenge, which aims to connect blockchain to a challenge-based trust mechanism. Challenge-based trust mechanisms measure a node's integrity by assessing the relationship between the sent challenges and the responses received. Traditional challenge-based mechanisms are effective for internal attacks but are insufficient for advanced attacks. The assessment is carried out for both random 
poisoning and special on-off attack (SOOA). The results show that the framework improves the resilience of the CIDN in terms of trust management.

\section{Proposed Work}

The proposed framework aims to find a different combination of a classification algorithm for different services that provide the best performance. The proposed work is divided into two parts: Blockchain Framework and IDS Modules used as participants in the network:

\subsection{Blockchain Framework}

In this section, the architecture of the proposed collaborative IDS model and its design principles are presented. To enhance the IDS collaborative framework's detection capabilities, IDS can share the required information with its peer nodes. The proposed framework provides a secure path of communication to different isolated IDS to share resources and information. Blockchain is used to provide a secure and trustful interaction between the IDSs. Blockchain is considered as distributed data structure that allows different entities connected to the network to share and verify the information without any mediator's participation. In other words, it is a decentralized ledger that can be used to record transactions on different participating nodes, and that protects data integrity with powerful cartographic tools.

Figure 1 represents the Collaborative IDS network framework, depicting blockchain's high-level architecture. There are two essential components used to connect IDSs with blockchain network are Blockchain management component and Blockchain collaboration component.

\subsubsection{Blockchain Management Component}

This component is responsible for maintaining and validating the blockchain and increasing the robustness of trust management by connecting nodes to a blockchain, i.e., sending information, voting, and obtaining decisions. In case if two nodes has different blockchain then longest chain is considered as valid if both are of same length then voting is performed among nodes. It is also responsible for establishing communication with other IDS nodes and organizing and administrating the trust among IDS nodes. It is used to verify the authenticity of the user by using a challenge-based approach so that malicious nodes cannot act as legitimate nodes. In this approach a problem or challenge is send to the node which is trying to access the resources from blockchain, this problem is designed in such a way that only a legitimate user can answer them if answer is incorrect that node cannot access the resources. It is also responsible for evaluating the reputation of other nodes using a certain trust approach. The node's higher reputation indicates that the data or information shared by that node is more reliable. 

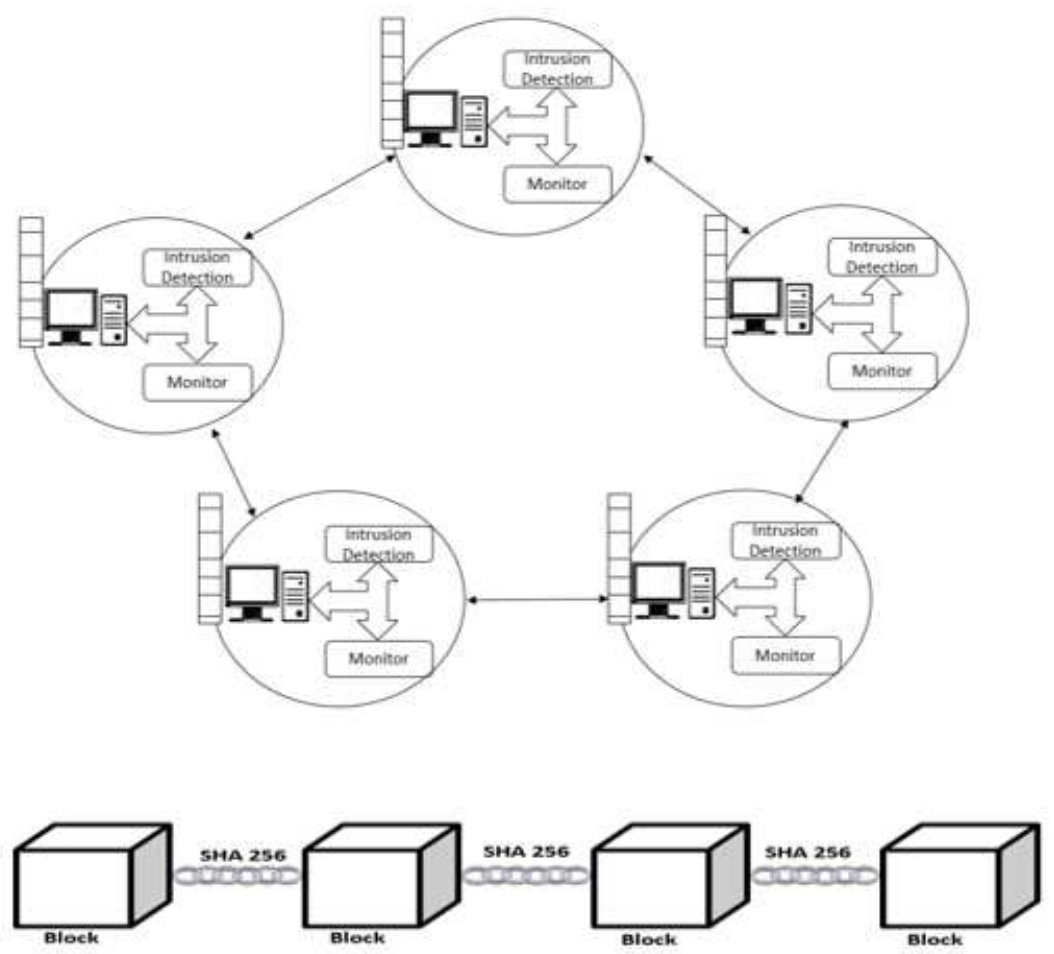

Fig. 1 Architecture of CIDS using blockchain

\subsubsection{Blockchain Collaboration Component}

Collaborative component is responsible for keeping track of new information and models. It also checks whether the information shared by particular nodes is useful or not. This component is also responsible for allocating or charge etherium points for every information transmitted or used by the nodes in the blockchain.

The proposed framework is used to enhance the IDS nodes' overall performance in the blockchain network by training different models on each IDS using their personalized dataset. The testing set is common for all IDS systems created by combining each IDS node's testing dataset to get the results that can be easily compared. This is also useful to test the IDS on a wide range of attacks and new attacks which may not be present in the current dataset of the IDS. After training is completed, the IDS model is sent to the peer management component, where the model's performance is checked and compared with the existing model on the blockchain. If the performance is greater than the existing model, a new block is created containing the new model, timestamp, performance, and previous hash. Hashing is done by using the SHA256 algorithm. The trained model is then compared with the previously stored model on blockchain as shown in Algorithm 1 where the new model is the ensemble model of a particular IDS module and prev model, which has the best performance at the given timestamp. If any model gives better performance, it is replaced, and the new timestamp is updated. To replace 
the model, we need to add a new block in blockchain with a new timestamp and information related to the block as shown in Algorithm 2. Before actually adding a block with the new model in blockchain, it has to be assigned with a new hash and provide proof of work $(\mathrm{PoW})$ as PoW is necessary to avoid frauds and enable trust about the model's genuineness in its peers.
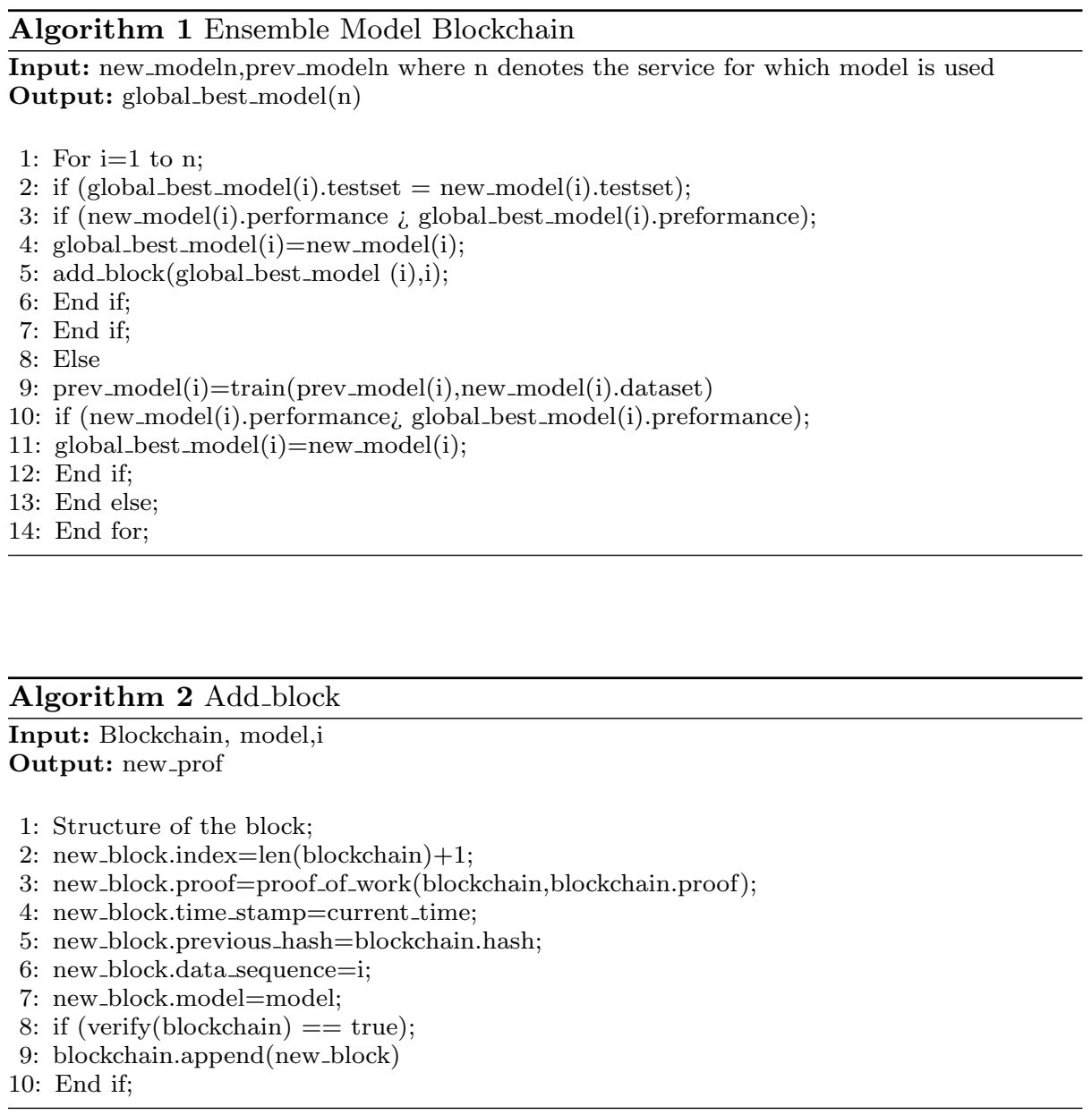

\subsection{IDS Module Architecture}

In the IoT environment, there are different types of devices connected to the network providing other services. It is difficult for a single model to detect attacks on such heterogeneous devices. For this purpose, different ensemble models are trained in the proposed work for detecting attacks on various services. It is also easier to compare and combine the ensemble model with other IDS modules with service-based training. As the proposed IDS framework is an ensemble learners 

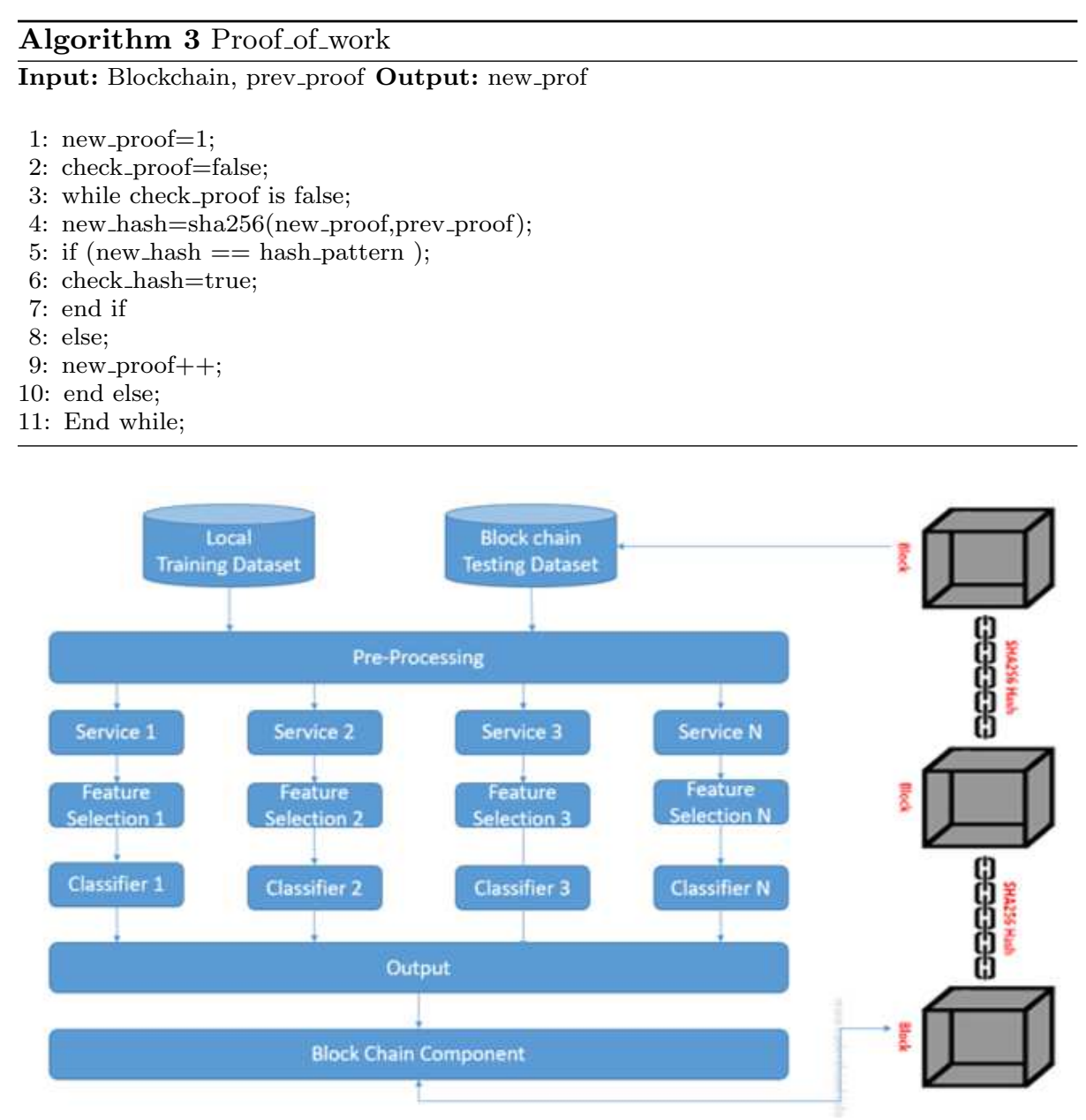

Fig. 2 IDS architecture with blockchain

module, each service /device can have different feature selection and classification algorithms. In the proposed IDS framework, Mutual Information Gain (MIG) algorithm, and the different classifiers are used, such as ANN, RF, and SVM. The workflow of the IDS modules is shown in Figure 2. At every IDS module, models are trained using a local training dataset, while the testing dataset is the combination of the shared test dataset of every module. The essential steps for training the model given as:1)Preprocessing 2)Feature selection 3)Classification 4)Ensemble Model.

\subsubsection{Preprocessing}

Before training the machine learning model, data is preprocessed to avoid errors, and invalid results as the dataset may contain some erroneous values or null values that need to be removed. It is also necessary to convert symbolic values to numer- 
ical values and normalized the continuous numerical datatype. It is also easier to compare and combine the ensemble model with other IDS modules with servicebased training. To evaluate the proposed method UNSW_NB15 dataset original files are used. Since there is no dataset which can be divided among 5 nodes so entire UNSW_NB15 csv file is used along with UNSW_NB15_1, UNSW_NB15_2, UNSW_NB15_3, UNSW_NB15_4 and each file of dataset is assigned to each IDS node. After that for service based classification dataset is grouped according to services. The services used in this case are DNS, HTTP and FTP on each IDS node. Each of the group is executed separately as shown in Figure 2. The reason behind grouping the data according to service is to improve detectability of anomaly in that particular service, this is useful to enhance performance when this different classifiers are trained on particular service and then ensemble. This also solves the problem of creating a common Benign traffic profile for large and diverse traffic as it is now grouped and classified according to services. In the proposed work, One hot encoding with min-max normalization is used for data preprocessing. Algorithm 1 shows the steps used to preprocess the dataset. Features containing nominal data are converted into binary values using one-hot encoding. Consider the 'Service' feature having nominal data DNS, HTTP, and FTP. This nominal data is converted into columns, and rows containing the data are considered 1 and remaining as 0 ; for example, $(0,0,1)(0,1,0)(1,0,0)$ will be the values for DNS, HTTP, and FTP, respectively. One-hot encoding divides a column of categorical data into multiple columns called dummy columns based on the number of categories in that column. Each column contains 0 or 1 , according to the column in which it is placed. It is very effective to compare to label encoding because in label encoding, we assign numerical value. Considering the same example of DNS, HTTP, and FTP with label encoding assigned values as DNS 1, HTTP as 2, and FTP as 3, this may confuse the machine learning model by adding DNS HTTP we can get FTP $(1+2=3)$. Once we convert the symbolic values to numerical values using one-hot encoding, we need to apply min-max normalization on the dataset to remove the continuous numerical data. The dataset variables range at different scales do not contribute equally to the model training, and the model might become bias towards particular features. The formula used for Min-Max scaling is:

$$
X_{n e w}=\frac{X_{i}-X_{\operatorname{Min}(X)}}{\operatorname{Max}(X)-\operatorname{Min}(X)}
$$

Here $\mathrm{X}$ is the feature that needs to be scaled, $\min (\mathrm{X})$ and $\max (\mathrm{X})$ are minimum and maximum values of feature respectively. $X_{i}$ is the current value being processed and $X_{n e w}$ will be the new value.

\subsubsection{Feature Selection}

The proposed architecture of the IDS Module is an ensemble learner in which each service data is considered as a different device in IoT network. Different IoT devices may have different data, working and attack patterns. The feature selection and classification of these devices are done using an ensemble model shown in IDS module Figure 2. As it is an ensemble model, different can have a different combination of Feature selection and classification algorithm. In the proposed 
framework, Mutual Information gain algorithm is used as feature selection for all the services.

Information Gain (IG) uses the entropy-based method for evaluating features, which is widely used in machine learning. Information Gain is defined as the amount of information provided by the feature contributing to decision-making. The information gain is calculated based on the part of the term that can be used to classify information to measure the importance of lexical elements for classification [1].

\subsubsection{Classification}

In the classification step, different machine learning classifiers are trained for every service to get the best possible model using data available by that particular IDS module. In the proposed work, two machine learning algorithms are used for classification, namely ANN and RF.

Artificial Neural Network(ANN): The ANN model trained for intrusion detection is a dense model with one input and one output layer densely connected with four hidden layers. The number of neurons in the input layer depends on the number of features selected during the feature selection algorithm. All the hidden layers contain 32 neurons, which are densely connected with ReLu as an activation function. The output layer contains only one neuron; as the proposed framework is a binary classifier, it needs to provide only two values, i.e., normal or anomaly traffic. Adam's optimization algorithm is used to maintain the neural network's weights and learning rate to reduce the losses during the training of the model, and losses are calculated using the binary cross-entropy function. The working of the Adam optimizer is shown in Algorithm 4 and the binary cross-entropy function is given in $\mathrm{Eq}(2)$.

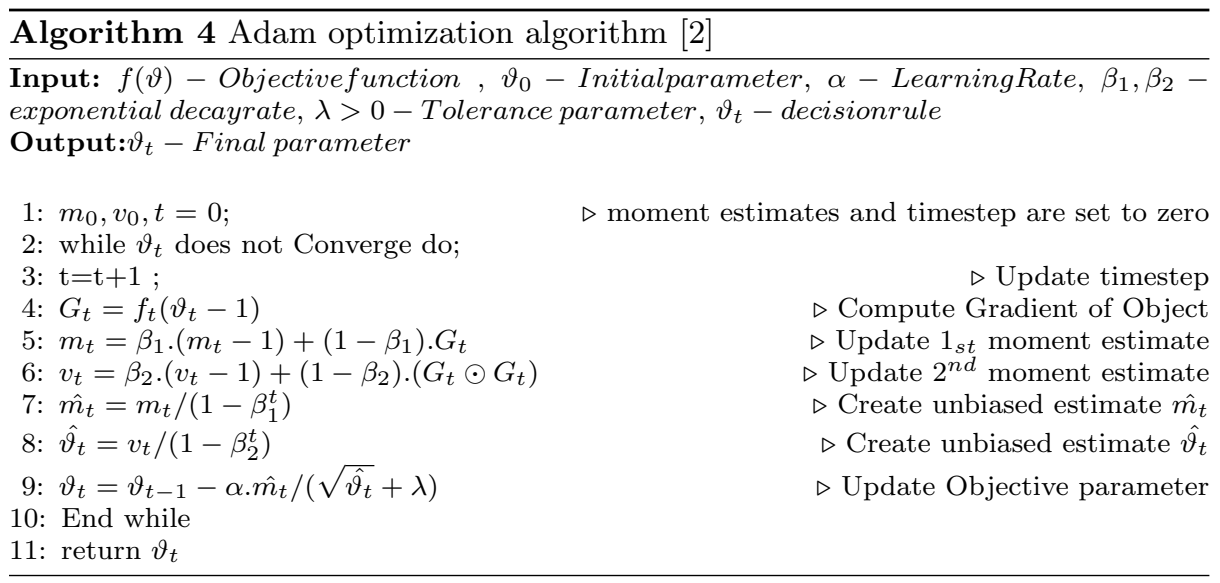

In Eq.(2), y represents the lables for the given data and $\mathrm{N}$ represents the number of classes. 


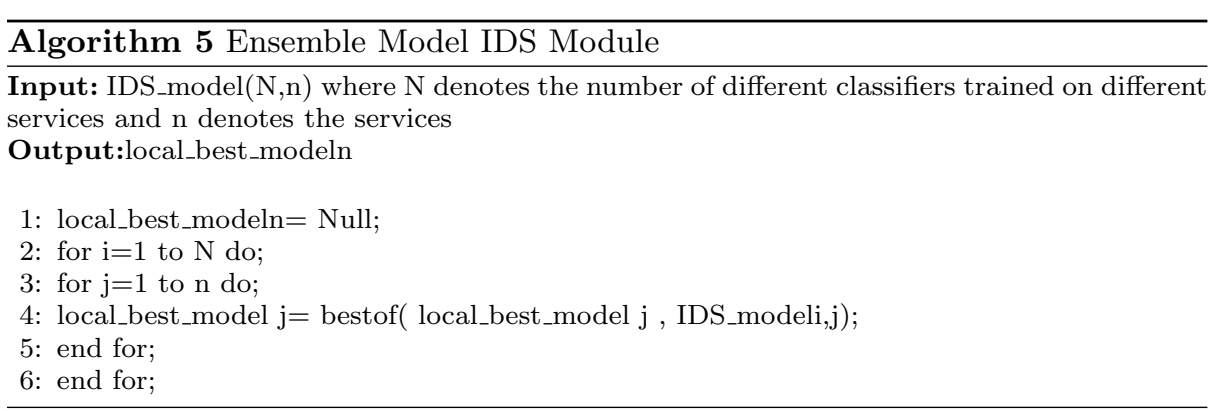

$$
H_{p}(q)=-\frac{1}{N} \Sigma_{i=1}^{N} y_{i} \cdot \log \left(p\left(y_{i}\right)\right)+\left(1-y_{i}\right) \cdot \log \left(1-p\left(y_{i}\right)\right)
$$

Random Forest-Random forest is an ensemble learning method that decides by voting using multiple decision trees. A decision tree is the building block of the random forest, which gives a build by determining the node's usefulness and then splits accordingly and eventually leads to the final classification. Gini index is used to calculate the contribution of a node in the decision, which is the probability that a randomly chosen sample in a node would be incorrectly labeled. If it was labeled by the distribution of samples in the node.

$$
I_{G}(n)=1-\Sigma_{i=1}^{J}\left(P_{i}\right)^{2}
$$

Here, $\mathrm{n}$ determines the current node or feature in the decision tree for which the gini impurity is to be calculated and $\mathrm{J}$ represents the number of classes to be classified in this case 2 (Normal and Anomaly)

\subsubsection{Ensemble Model IDS Module}

In this step, the performance of different IDS module models is compared individually for various services. After comparing the models, a single ensemble model is created by selecting the best performance model for each service currently present locally at the IDS module. This process is described in Algorithm 5 which gives a set of local models as output called as local_best_model; this set is then compared with the global model present on the blockchain.

\section{Result analysis}

The implementation and evaluation of proposed work are done using Python 3.7, and different blockchain nodes are executed using Postman API on Dell system on Windows 10, with a processor of 64 bit, RAM of 8 GB with Intel(R) Core(TM) i5-7200U, CPU2.50 GHz. UNSW_NB15 intrusion detection dataset [34,35] is used to validate the proposed framework.

The proposed work is a collaborative IDS framework using blockchain in which each IDS module can train different machine learning models. To mimic this, five IDS modules are trained, namely IDS module A, B, C, D, and E, respectively. Each module has its own training dataset, while the complete training data set is 
Table 1 Performance of different ML model trained on each IDS Module for given test dataset

\begin{tabular}{|c|c|c|c|c|c|c|}
\hline $\begin{array}{l}\text { Service/ } \\
\text { Device }\end{array}$ & Model & $\begin{array}{l}\text { IDS } \\
\text { Module }\end{array}$ & Accuracy & Recall & Precision & Fscore \\
\hline \multirow{10}{*}{ DNS } & \multirow{5}{*}{ ANN } & $\mathrm{A}$ & 58.603 & 99.94 & 58.54 & 0.7384 \\
\hline & & B & 62.915 & 99.96 & 61.18 & 0.7591 \\
\hline & & $\mathrm{C}$ & 73.453 & 99.99 & 68.77 & 0.8149 \\
\hline & & $\mathrm{D}$ & 59.089 & 99.98 & 58.82 & 0.74 \\
\hline & & $\mathrm{E}$ & 99.612 & 99.99 & 99.35 & 0.9966 \\
\hline & \multirow{5}{*}{$\mathrm{RF}$} & $\mathrm{A}$ & 91.816 & 99.69 & 87.9 & 0.9343 \\
\hline & & $\mathrm{B}$ & 99.655 & 99.77 & 99.63 & 0.997 \\
\hline & & $\mathrm{C}$ & 99.762 & 99.95 & 99.63 & 0.9979 \\
\hline & & $\mathrm{D}$ & 94.676 & 85.56 & 99.98 & 0.9221 \\
\hline & & $\mathrm{E}$ & 94.676 & 85.56 & 99.98 & 0.9221 \\
\hline \multirow{10}{*}{ HTTP } & \multirow{5}{*}{ ANN } & $\mathrm{A}$ & 96.836 & 96.94 & 99.11 & 0.9801 \\
\hline & & B & 97.589 & 97.95 & 99.04 & 0.9849 \\
\hline & & $\mathrm{C}$ & 96.877 & 98.77 & 97.39 & 0.9807 \\
\hline & & $\mathrm{D}$ & 89.373 & 99.54 & 88.68 & 0.9379 \\
\hline & & $\mathrm{E}$ & 97.46 & 98.88 & 97.98 & 0.9843 \\
\hline & \multirow{5}{*}{$\mathrm{RF}$} & $\mathrm{A}$ & 96.295 & 97.44 & 97.95 & 0.9769 \\
\hline & & B & 95.558 & 99.52 & 95.18 & 0.9731 \\
\hline & & $\mathrm{C}$ & 97.489 & 99.04 & 97.87 & 0.9845 \\
\hline & & $\mathrm{D}$ & 86.942 & 99.78 & 86.21 & 0.925 \\
\hline & & $\mathrm{E}$ & 96.481 & 98.85 & 96.84 & 0.9784 \\
\hline \multirow{10}{*}{ FTP } & \multirow{5}{*}{ ANN } & $\mathrm{A}$ & 98.553 & 99.99 & 98.54 & 0.9926 \\
\hline & & $\mathrm{B}$ & 98.429 & 100 & 98.41 & 0.992 \\
\hline & & $\mathrm{C}$ & 98.478 & 99.99 & 98.46 & 0.9922 \\
\hline & & $\mathrm{D}$ & 99.171 & 99.98 & 99.17 & 0.9957 \\
\hline & & $\mathrm{E}$ & 99.989 & 99.99 & 99.99 & 0.9999 \\
\hline & \multirow{5}{*}{$\mathrm{RF}$} & $\mathrm{A}$ & 99.73 & 99.77 & 99.94 & 0.9986 \\
\hline & & $\mathrm{B}$ & 99.695 & 99.8 & 99.88 & 0.9984 \\
\hline & & $\mathrm{C}$ & 99.973 & 99.99 & 99.98 & 0.9998 \\
\hline & & $\mathrm{D}$ & 99.801 & 99.84 & 99.94 & 0.9989 \\
\hline & & $\mathrm{E}$ & 99.818 & 99.88 & 99.93 & 0.999 \\
\hline
\end{tabular}

built by collecting training data from each module. The benefit of using a common testing data set is that some of the attack patterns may not be available to other IDS modules. It is easy to compare the performance of different IDS Modules.

UNSW_NB15 dataset is used to evaluate the performance. The UNSW_NB15 dataset divided five datasets, and each dataset is passed to a different IDS module. The dataset contains 42 features, and the dataset is grouped according to the 


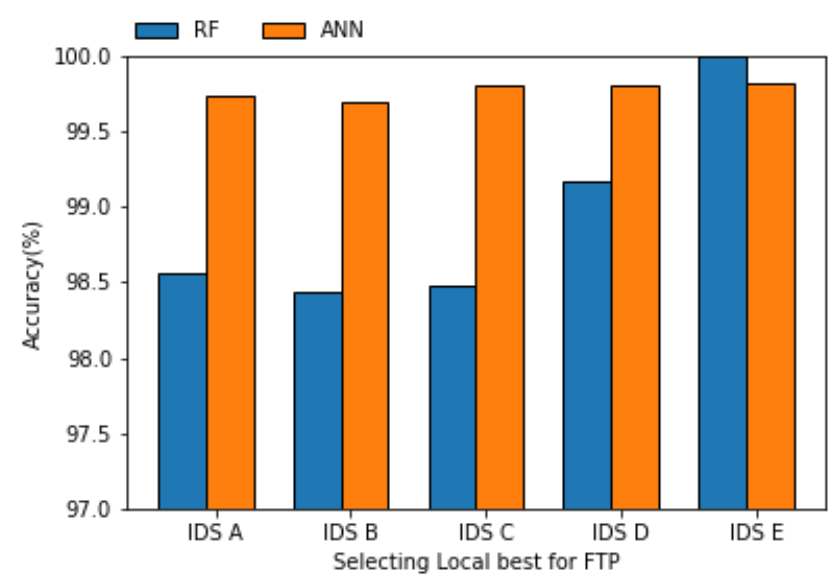

Fig. 3 Selecting best model in blockchain for FTP service

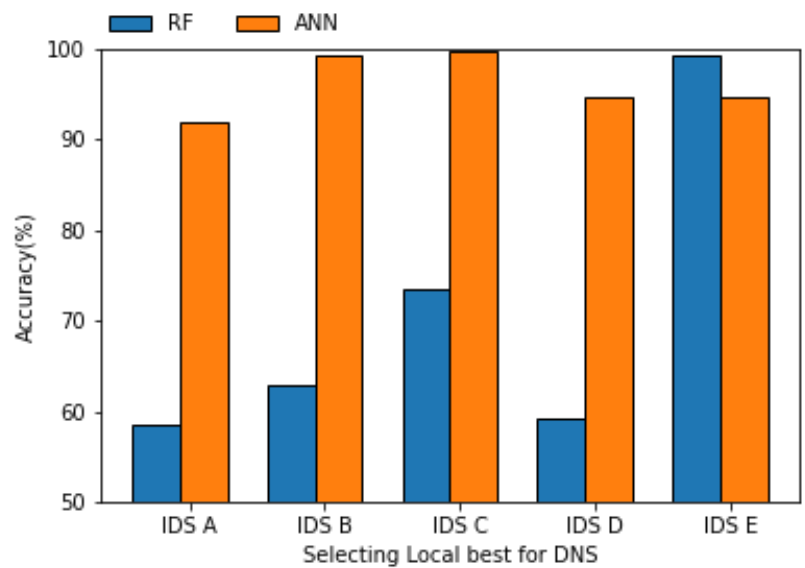

Fig. 4 Selecting best model in blockchain for DNS service

different attacks that occurred in different services, which are assumed as different IoT devices in the experimentation. The dataset contains nine categories of attacks, i.e., fuzzers, analysis, backdoors, DoS, exploits, generic, reconnaissance, shellcode, and worms.

In the experimentation series, each IDS model is trained according to the steps mentioned in section 3.2. Before training the model, the dataset needs to be cleaned and preprocessed as it may contain some errors, null values, and symbolic values. The records containing null values and errors are either deleted or replaced with value 0 . The symbolic values are converted into binary values by using dummy columns with one hot encoding method.

After performing One hot encoding on numerical data, normalization is performed on ranged numerical data to bring the values on a common scale. Minmax normalization technique is used for this purpose; without this, the model might 


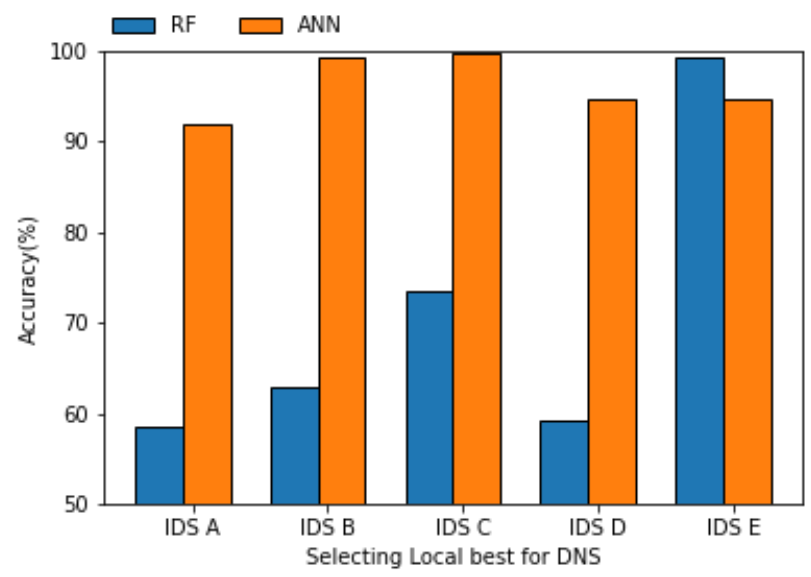

Fig. 5 Selecting best model in blockchain for HTTP service

produce bias results based on larger values. After preprocessing, features are selected based on information gain. After feature selection, data is sent to the machine learning model and trained for each service as here different services are used to mimic different IoT devices. All IDS models are trained with random forest and ANN, as proposed framework is for ensembling different machine models and it can be combined with any other machine learning model.

The performance of individual IDS modules for RF and ANN model is shown in Table 1. The Local best model is selected by comparing the performance of the model trained for that particular service. Consider the example of IDS module A here for DNS service. Two models are trained; one is RF which has $58.603 \%$ accuracy, and another is ANN which has 91.816\% accuracy. Hence for DNS service in the IDS module, A ANN model is selected. Similar steps are followed for all the services and remaining IDS modules. For example, consider IDS Module B DNS service has $99.65 \%$ accuracy with ANN. HTTP has $97.589 \%$ accuracy with RF and FTP has $99.69 \%$ accuracy with ANN, making the total performance of local ensemble mode as $98.979 \%$. The process of selecting the local best model for the IDS module is given in Algorithm 4. After finding the local best models, each IDS module compares the performance with the globally best model present on the blockchain.

The process of selecting the globally best model is done by following Algorithm 1. Figure 3, Figure 4 and Figure 5 graphically represent the selection of the globally best model that has to be added in the blockchain. In Figure 3 IDS module $\mathrm{C}$ with ANN has the best accuracy of $99.762 \%$ for DNS service, similarly for HTTP and FTP in Figure 4 and 5 IDS module B with RF and IDS E with RF module has the best accuracy of $97.589 \%$ and $99.989 \%$ respectively. This model is added to the blockchain by creating a new block as shown in Algorithm 2. This selected model CEBM on the blockchain is created by ensembling different modules.

The performance of the proposed framework CEBM is shown in Table 2. Figure 6 shows the comparison of the performance of IDS modules and CEBM. The overall performance of the CEBM outperforms the performance of the individual IDS 
Table 2 Performance of collaborative model selected in blockchain

\begin{tabular}{|l|r|r|r|r|}
\hline Service & Accuracy(\%) & Recall & Precision & Fscore \\
\hline DNS & 99.762 & 99.958 & 99.635 & 0.9979 \\
\hline HTTP & 97.589 & 97.955 & 99.048 & 0.9849 \\
\hline FTP & 99.989 & 99.992 & 99.996 & 0.9999 \\
\hline Total & $\mathbf{9 9 . 1 1 3}$ & $\mathbf{9 9 . 1 7 7}$ & $\mathbf{9 9 . 5 5 9}$ & $\mathbf{0 . 9 9 4 2}$ \\
\hline
\end{tabular}

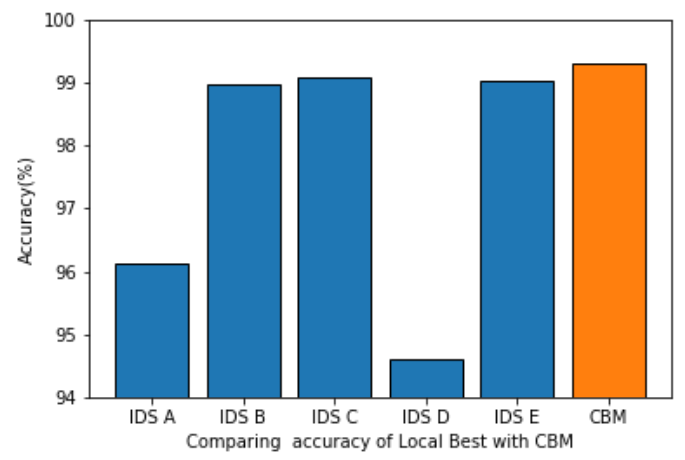

Fig. 6 Accuracy comparison of local best models with CEBM

Modules with $99.113 \%$ accuracy. In the proposed framework each node present in Figure 1 is an IDS and each IDS is trained as shown in Figure 2. Thus in this example each node will train on 3 services HTTP, FTP and DNS using its own dataset to train and blockchain testing dataset which is common for all nodes to create unbiased results. The performance of each node for each service is shown in Figure 6. Since each node contains different part of UNSW_NB15 dataset it is very difficult to compare the proposed approach with traditional IDS.

Along with comparing CEBM with individual IDS modules, the proposed work is also compared with the recent state-of-the-art models as shown in Table 3. It is observed that the proposed framework outperformed the current state of art models by achieving $99.113 \%$ accuracy. The visual representation of the comparison of the proposed framework accuracy with the state the art model is shown in Figure 7. CEBM is the proposed framework that outperforms other existing state-of-the-art approaches.

\subsection{Discussion}

The service base training of classifier shows an excellent performance as devices connected to a network uses many services of network sometime an intruder may attack through some file transfer or mail or through some other domain for example HTTP, FTP and DNS services. Due the growth of diverse IoT devices in IoT network it is very difficult for create a benign traffic profile for all this devices and services to detect anomaly instead a novel approach for service based grouping before feature selection and classification is applied as shown in Figure 2. It helps 
Table 3 Performance comparison of CEBM with present state of art models

\begin{tabular}{|c|c|c|c|c|c|c|}
\hline Sr. & Model & Dataset & Accuracy & Recall & Precision & F-Score \\
\hline 1 & $\begin{array}{l}\text { Trapezoidal area estima- } \\
\text { tion }[28]\end{array}$ & UNSW & 91.8 & 91 & - & - \\
\hline 2 & Finite Dirichlet model [29] & UNSW & 94.3 & 93.9 & - & - \\
\hline 3 & $\mathrm{GA}+\mathrm{DT}[30]$ & UNSW & 82.42 & - & - & - \\
\hline 4 & Gottwalt [31] & UNSW & 98.65 & 99.74 & - & - \\
\hline 5 & FFDNN [32] & UNSW & 87.48 & - & - & - \\
\hline 6 & TSE-IDS [36] & UNSW & 91.27 & - & 91.6 & - \\
\hline 7 & Proposed framework** & UNSW & 99.113 & 99.177 & 99.559 & 0.9942 \\
\hline
\end{tabular}



Fig. 7 Accuracy comparison of CEBM with present state of the art models

to simplify the problem of creating a common benign traffic profile for such large and diverse data and also is useful to enhance performance. This is the working of first part of proposed framework i.e., working of each node shown in Figure 1. In this experimentation each node in Figure 1 namely A, B, C, D, E is trained for 3 services FTP, HTTP and DNS and each service is trained on different classifier as shown in Figure 2. Blockchain is use to collaborate these nodes to enhance the performance of IDS by ensemble best model of each service as shown in Table 2. The ensemble model is created by comparing performance of each IDS for that particular service and an ensemble model with best performance is created and shared using block chain. For example, Table 1 shows the performance of all the nodes for all three services, consider service DNS if a particular model has poor performance in detecting anomaly for DNS service due to lack of DNS data or poisoning it can be improved by comparing to the best model for DNS in collaborative environment i.e., DNS-RF-C (Module $\mathrm{C}$ using $\mathrm{RF}$ has best performance for service DNS of $99.762 \%$ ). Similar by ensembling best model of each service using Blockchain a collaborative ensemble Blockchain model is created which has the best performance compare to all nodes inn network. This is helpful to improve the performance of IDS that has poor performance due to poisoning during trading or suffer from lack of data to train. 


\section{Conclusion}

IDS is an essential component of the security framework to create a secure IoT environment. But an isolated or individual IDS module used for IoT devices has its limitation and may suffer from a lack of information. The proposed work aims to provide a generic collaborative framework where different IDS modules can share their knowledge to boost intrusion detection's overall performance. The performance of the ensemble model created by collaborating with different IDS modules is greater than individual module performance. For example, the accuracy of the Collaborative IDS model is $99.113 \%$, and the accuracy of the IDS module is $96.125 \%$, therefore with the help of the proposed framework, the accuracy of the IDS module $2.988 \%$ can boost a. If we look at the performance of individual services, the performance of DNS is increased from $91.8 \%$ to $99.7 \%$. Hence, from the experimentation, the IDS model's performance can be improved with the help of a collaborative network, and this collaborative network can be secured using blockchain technology.

\section{Declerations}

Acknowledgement Authors gratefully thank the Maulana Azad National Institute of Technology (MANIT) for their support and resource facilities required in carrying out the research reported here.

Funding Research presented in the manuscript are not funded by any external agencies.

Conficts of Interest The authors declare no confict of interest.

\section{References}

1. Otoum Y, Liu D, Nayak A. DL-IDS: a deep learning-based intrusion detection framework for securing IoT. Transactions on Emerging Telecommunications Technologies. 2019 Nov 29:e3803.

2. Mirsky Y, Golomb T, Elovici Y. Lightweight collaborative anomaly detection for the IoT using blockchain. Journal of Parallel and Distributed Computing. 2020 Nov 1;145:75-97.

3. Khan R, Khan SU, Zaheer R, Khan S. Future internet: the internet of things architecture, possible applications and key challenges. In2012 10th international conference on frontiers of information technology 2012 Dec 17 (pp. 257-260). IEEE.

4. Balasubramanian V, Zaman F, Aloqaily M, Al Ridhawi I, Jararweh Y, Salameh HB. A mobility management architecture for seamless delivery of 5G-IoT services. InICC 2019-2019 IEEE International Conference on Communications (ICC) 2019 May 20 (pp. 1-7). IEEE.

5. Al Ridhawi I, Kotb Y, Aloqaily M, Jararweh Y, Baker T. A profitable and energy-efficient cooperative fog solution for IoT services. IEEE Transactions on Industrial Informatics. 2019 Jun 17;16(5):3578-86.

6. Abbas N, Asim M, Tariq N, Baker T, Abbas S. A mechanism for securing IoT-enabled applications at the fog layer. Journal of Sensor and Actuator Networks. 2019 Mar;8(1):16.

7. Kotb Y, Al Ridhawi I, Aloqaily M, Baker T, Jararweh Y, Tawfik H. Cloud-based multiagent cooperation for IoT devices using workflow-nets. Journal of Grid Computing. 2019 Dec;17(4):625-50.

8. oT: Number of Connected Devices Worldwide 2012-2025," Statista. [Online]. Available: https://www.statista.com/statistics/471264/iot-number-of-connected-devices worldwide/. 
9. Rathore S, Kwon BW, Park JH. BlockSecIoTNet: Blockchain-based decentralized security architecture for IoT network. Journal of Network and Computer Applications. 2019 Oct $1 ; 143: 167-77$.

10. Scarfone K, Mell P. Guide to intrusion detection and prevention systems (idps). NIST special publication. 2007 Feb 20;800(2007):94.

11. Bhattacharya S, Maddikunta PK, Kaluri R, Singh S, Gadekallu TR, Alazab M, Tariq U. A novel PCA-firefly based XGBoost classification model for intrusion detection in networks using GPU. Electronics. 2020 Feb;9(2):219.

12. Alazab M, Khan S, Krishnan SS, Pham QV, Reddy MP, Gadekallu TR. A multidirectional LSTM model for predicting the stability of a smart grid. IEEE Access. 2020 Apr 28;8:8545463.

13. Iwendi C, Maddikunta PK, Gadekallu TR, Lakshmanna K, Bashir AK, Piran MJ. A metaheuristic optimization approach for energy efficiency in the IoT networks. Software: Practice and Experience. 2020 Feb 11.

14. Khan S, Kifayat K, Kashif Bashir A, Gurtov A, Hassan M. Intelligent intrusion detection system in smart grid using computational intelligence and machine learning. Transactions on Emerging Telecommunications Technologies. 2020 Aug 3:e4062.

15. Li D, Cai Z, Deng L, Yao X, Wang HH. Information security model of block chain based on intrusion sensing in the IoT environment. Cluster computing. 2019 Jan;22(1):451-68.

16. Li D, Liu W, Deng L, Qin B. Design of multimedia blockchain privacy protection system based on distributed trusted communication. Transactions on Emerging Telecommunications Technologies. 2020 Apr 5.

17. Meng W, Tischhauser EW, Wang Q, Wang Y, Han J. When intrusion detection meets blockchain technology: a review. Ieee Access. 2018 Jan 30;6:10179-88.

18. Golomb T, Mirsky Y, Elovici Y. CIoTA: Collaborative IoT anomaly detection via blockchain. arXiv preprint arXiv:1803.03807. 2018 Mar 10.

19. Nakamoto S. Bitcoin: A peer-to-peer electronic cash system. Manubot; 2019 Nov 20.

20. Wood G. Ethereum: A secure decentralised generalised transaction ledger. Ethereum project yellow paper. 2014 Apr;151(2014):1-32

21. Meng W, Tischhauser EW, Wang Q, Wang Y, Han J. When intrusion detection meets blockchain technology: a review. Ieee Access. 2018 Jan 30;6:10179-88.

22. Li W, Tug S, Meng W, Wang Y. Designing collaborative blockchained signature-based intrusion detection in IoT environments. Future Generation Computer Systems. 2019 Jul 1;96:481-9.

23. Meng W, Li W, Yang LT, Li P. Enhancing challenge-based collaborative intrusion detection networks against insider attacks using blockchain. International Journal of Information Security. 2019 Aug 8:1-2.

24. Manikumar DV, Maheswari BU. Blockchain Based DDoS Mitigation Using Machine Learning Techniques. In2020 Second International Conference on Inventive Research in Computing Applications (ICIRCA) 2020 Jul 15 (pp. 794-800). IEEE.

25. Li W, Wang Y, Li J, Au MH. Toward a blockchain-based framework for challenge-based collaborative intrusion detection. International Journal of Information Security. 2020 Feb 11:1-3.

26. Lei S. A feature selection method based on information gain and genetic algorithm. In2012 International Conference on Computer Science and Electronics Engineering 2012 Mar 23 (Vol. 2, pp. 355-358). IEEE.

27. Baydin AG, Pearlmutter BA, Radul AA, Siskind JM. Automatic differentiation in machine learning: a survey. Journal of machine learning research. 2018 Apr 1;18.

28. Moustafa N, Slay J, Creech G. Novel geometric area analysis technique for anomaly detection using trapezoidal area estimation on large-scale networks. IEEE Transactions on Big Data. 2017 Jun 14;5(4):481-94.

29. Moustafa N, Creech G, Slay J. Big data analytics for intrusion detection system: Statistical decision-making using finite dirichlet mixture models. InData analytics and decision support for cybersecurity 2017 (pp. 127-156). Springer, Cham.

30. Khammassi C, Krichen S. A GA-LR wrapper approach for feature selection in network intrusion detection. computers \& security. 2017 Sep 1;70:255-77.

31. Gottwalt F, Chang E, Dillon T. CorrCorr: A feature selection method for multivariate correlation network anomaly detection techniques. Computers \& Security. 2019 Jun 1;83:23445.

32. Kasongo SM, Sun Y. A deep learning method with wrapper based feature extraction for wireless intrusion detection system. Computers \& Security. 2020 May 1;92:101752. 
33. Verma P, Tapaswi S, Godfrey WW. AVDR: A Framework for Migration Policy to Handle DDoS Attacked VM in Cloud. Wireless Personal Communications. 2020 Nov;115(2):1335-61. 34. Moustafa N, Slay J. UNSW-NB15: a comprehensive data set for network intrusion detection systems (UNSW-NB15 network data set). In2015 military communications and information systems conference (MilCIS) 2015 Nov 10 (pp. 1-6). IEEE.

35. https://www.unb.ca/cic/datasets/nsl.html

36. Tama BA, Comuzzi M, Rhee KH. TSE-IDS: A two-stage classifier ensemble for intelligent anomaly-based intrusion detection system. IEEE Access. 2019 Jul 11;7:94497-507.

37. Verma P, Tapaswi S, Godfrey WW. A request aware module using CS-IDR to reduce VM level collateral damages caused by DDoS attack in cloud environment. Cluster Computing. 2021 Jan 23:1-7. 